\title{
Uso de placas de desarrollo para la mejora de la docencia en las prácticas de electrónica
}

\section{Use of development boards to improve teaching in the electronics lab}

JUAN ANTONIO LEÑERo BARDALLO

ORCID: https://orcid.org/0000-0002-1741-9743

Universidad de Sevilla

Departamento de Electrónica

y Electromagnetismo

jlenero@us.es

Fecha de recepción: 08-06-2019.

Fecha de aceptación: 25-06-2019

DOI: http://dx.doi.org/10.12795/9788447221912.028 Pp.: 647-664 
Las prácticas de electrónica requieren el análisis teórico previo de un circuito que se va a caracterizar, el montaje del mismo en una placa de pruebas, la configuración de los instrumentos del laboratorio, la toma de medidas y el análisis de medidas realizadas. El tiempo asignado a las sesiones prácticas impide que todo el proceso completo pueda llevarse a cabo en una única sesión de clase. Además, los alumnos de cursos iniciales carecen de la pericia necesaria para interpretar un diagrama eléctrico y montar el circuito descrito en una placa de pruebas. Esto hace que el proceso de montaje sea lento, necesite ser supervisado por el profesor y esté sujeto a posibles errores que limiten el resto de la práctica. Se evaluó mediante un Ciclo de Mejora en el Aula (CIMA) el uso en las sesiones de laboratorio de Placas de Desarrollo que permiten experimentar y tomar medidas directamente con varios circuitos de interés para la docencia.

Palabras clave: Electrónica Física, Grado en Física, docencia universitaria, experimentación docente universitaria, Placas de Desarrollo.

\section{Abstract}

The electronics lab lessons require the previous theoretical analysis of a circuit that is going to be characterized, the assembly of the same in a test board, the configuration of the laboratory instruments, and taking of measurements and the analysis of them. The time allocated to practical sessions prevents the entire process from being carried out in a single class session. In addition, students in initial courses lack the necessary expertise to interpret an electrical diagram and assemble the circuit described on a test board. This makes the assembly process slow, it needs to be supervised by the teacher and it is subject to possible errors that limit the rest of the session. It was evaluated through a Cycle of Improvement in the Classroom (CIMA) the use in the laboratory sessions of Development Boards that allow experimenting and taking measures directly with several circuits of interest for teaching

Key words: Physics electronics, Bachelor in Physics, university teaching, university teaching research, development boards. 


\section{Contexto de la intervención}

Las actividades de la innovación docente se desarrollaron en los seminarios prácticos de la asignatura Electrónica Física. Se trata de una asignatura troncal y obligatoria de seis créditos, que se imparte en el segundo cuatrimestre de forma simultánea en varias titulaciones: Grado en Física, el Grado en Ingeniería de Materiales y los dobles grados en Física y Matemáticas y en Física e Ingeniería de Materiales.

Los seminarios comprenden la realización de prácticas en el laboratorio. En ellas, los alumnos deben montar circuitos previamente explicados en clase y tomar medidas de los mismos. Las sesiones de seminarios tienen una duración reducida de unos 40 minutos efectivos. En muchos casos, el alumno dedica la mayor parte del tiempo al montaje de los circuitos bajo estudio, no disponiendo de tiempo suficiente para la toma de medidas antes de finalizar la clase. Ello genera estrés en el alumnado y en el profesorado, puesto que no se cumplen los objetivos propuestos.

Durante el curso 2018-2019, fruto de un proyecto de Innovación Docente (sol-201700083174-tra) con profesores de la Universidad de Cádiz, se montaron varias placas de circuitos impresos con circuitos que típicamente son usados para el aprendizaje en las sesiones prácticas de electrónica. De forma genérica, nos referiremos a ellas como ‘Placas de Desarrollo'. Fueron concebidas con la idea de evitar a los alumnos el montaje de tales circuitos, para que se pudieran centrar exclusivamente en la toma de medidas y el análisis de ellas.

Se pretende hacer uso de estas Placas de Desarrollo con la idea de que los alumnos ahorren tiempo y puedan tomar medidas experimentales de circuitos electrónicos que deben comprender, sin necesidad de hacer su montaje. 


\section{Descripción de las clases prácticas durante cursos anteriores}

En cursos anteriores las sesiones de prácticas de laboratorio que se han impartido en nuestro Departamento se hacían partiendo de un guion que el profesor proporcionaba con antelación. El alumno debía montar los circuitos propuestos en una placa de pruebas. Una vez hecho esto, pasaban a tomar las medidas que se indicaban en la memoria.

Aunque los alumnos deben aprender a realizar el montaje de circuitos a partir de un esquemático previo, eran muchos los alumnos que dedicaban la mayor parte del tiempo en el laboratorio a montar estos circuitos, no siéndoles posible la toma de medidas necesaria para entender la funcionalidad de los circuitos propuestos. Ante esta situación y la falta de tiempo para realizar el montaje y la toma de medidas en la asignatura de Electrónica Física, se propone usar placas de desarrollo con los circuitos montados, para que los alumnos puedan dedicarse en exclusiva a la toma de medidas en algunas de las sesiones de laboratorio clave para la asignatura.

El esquema de los seminarios de problemas seguía la siguiente secuencia en cursos anteriores:

1. Publicación del guion de prácticas en la Plataforma de Enseñanza Virtual

2. Preparación de la pizarra, presentación, e introducción de la materia a tratar en la sesión

3. Montaje de los circuitos en la placa de pruebas por parte de los alumnos.

4. Toma de medidas.

5. Discusión de los resultados con el profesor.

La secuencia habitual anterior se resume de la siguiente manera:

Planteamiento del problema + Montaje + Toma de medidas + Análisis de los resultados + Discusión con el profesor 


\section{Diseño previo del Ciclo de Mejora en el Aula}

\section{Modelo metodológico ideal}

El modelo metodológico ideal en las clases de laboratorio pretende que los alumnos realicen, por sí mismos, desde cero todas las tareas prácticas posibles, (Bain, 2007):

1. Los alumnos leen y preparan el guion disponible en Enseñanza Virtual, antes de ir al laboratorio.

2. El profesor hace una introducción sobre los objetivos de la práctica.

3. Los alumnos montan los circuitos propuestos.

4. Los estudiantes toman medidas de los circuitos propuestos.

5. Seguidamente, hacen un análisis crítico de los resultados.

6. Estos resultados son discutidos con el profesor y el resto de la clase.

\section{Modelo metodológico posible}

El modelo metodológico posible se ajusta a las limitaciones temporales existentes para realizar las prácticas durante el horario lectivo. Se pretende prescindir del montaje de los circuitos, que se proporcionó directamente a los alumnos en una placa de desarrollo. Se sacrificó pues el tiempo dedicado al montaje (típicamente unos $25 \mathrm{mi}$ nutos de media) para que los alumnos se centrasen en la toma de medidas y su análisis.

El desarrollo de las sesiones sigue la siguiente distribución de actividades, dentro del modelo metodológico posible (la sesión estándar es de 60 minutos de duración):

- 10 minutos. Introducción teórica. Preguntas clave y restos a resolver en el laboratorio. 
- 25 minutos. Toma de medidas por parte de los alumnos.

- 10 minutos. Análisis de las medidas obtenidas por parte de los alumnos.

- 10 minutos. Discusión de los resultados con el profesor.

El modelo posible incorpora, nuevos objetivos docentes dirigidos a:

- El análisis práctico de circuitos.

- Entender las limitaciones de los modelos utilizados en clase para analizar circuitos y la necesidad de realizar medidas para validarlos.

- Dominio de los instrumentos de medida disponibles en el laboratorio.

\section{Mapa de contenidos}

En la Figura 1, se muestra el mapa de contenidos que se trabajó en las sesiones prácticas de la asignatura. Estos son de tres tipos: conceptuales, procedimentales y actitudinales. Se muestran las dependencias entre ellos. Como se observa, el montaje de circuitos constituye un cuello de botella en el proceso de toma de medidas en el laboratorio. Si no se realiza correctamente, no es posible que el alumno adquiera las competencias de la asignatura.

En las sesiones del Ciclo de Mejora en el Aula (CIMA) que incorporen las Placas de desarrollo, el montaje de circuitos no será necesario, con la idea de facilitar que el alumno pueda asimilar los conceptos básicos de las sesiones.

Jornadas de Formación e Innovación Docente del Profesorado | № 2 (2019) Esta obra se distribuye con la licencia Creative Commons Reconocimiento-NoComercial-SinObraDerivada 


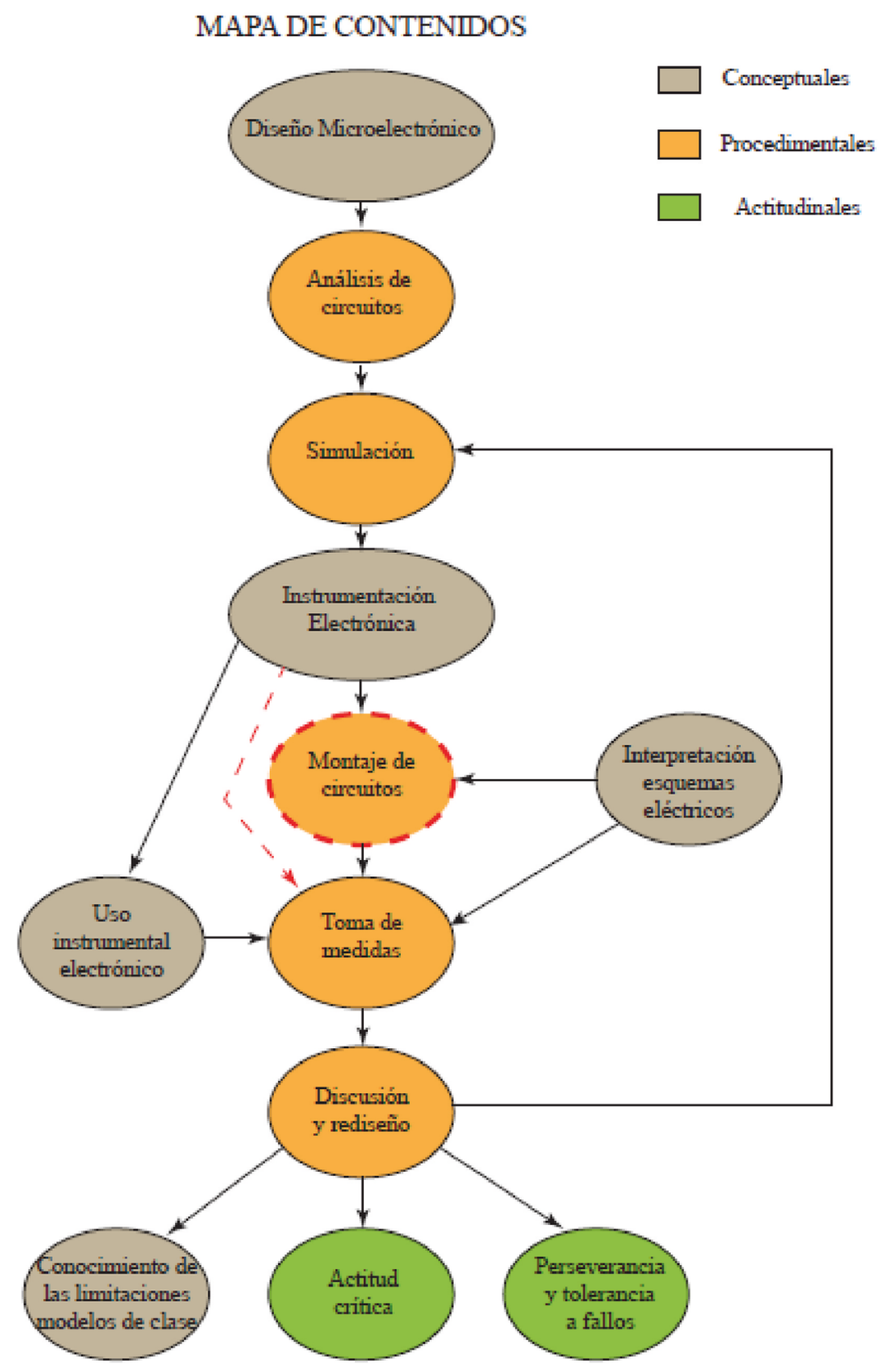

Figura 1. Mapa de contenidos asociados a las sesiones prácticas donde se realizó el Ciclo de Mejora en el Aula

Jornadas de Formación e Innovación Docente del Profesorado | № 2 (2019) Esta obra se distribuye con la licencia Creative Commons Reconocimiento-NoComercial-SinObraDerivada 4.0 Internacional (CC BY-NC-ND 4.0.) 
En la Figura 2, se detallan los contenidos específicos que se programaron para cada una de las sesiones:

El diodo de unión.
Efectos de segundo orden y desviaciones con respecto al
comportamiento ideal.
Circuitos con diodos: rectificadores de media onda y onda
completa

El transistor bipolar.

Efectos de segundo orden y desviaciones con respecto al comportamiento ideal.

Circuitos con transistores bipolares: circuito de polarización y amplificador de tensión.

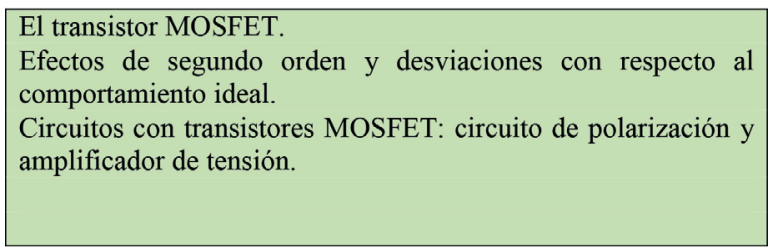

Circuitos con

diodos

( 8 sesiones)

Circuitos con transistores bipolares (7 sesiones)

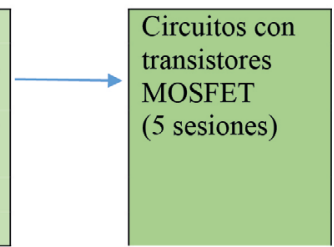

Figura 2. Diagrama con cada uno de los contenidos de cada una de las sesiones

\section{Secuencia de actividades del Ciclo de Mejora en el Aula}

Se detallan en la Tabla 1 la secuencia de actividades que se realizaron en cada una de las sesiones prácticas. Para cada una de estas actividades, se detalla el tipo de actividad que le corresponde.

Tabla 1. Secuencia de actividades del Ciclo de Mejora en el Aula

\begin{tabular}{|l|l|}
\hline № de actividad & \\
& $\begin{array}{l}\text { C: } \text { Cuestionario } \\
\text { TS: Tarea a realizar por el estudiante } \\
\text { TP: Tarea a realizar por el profesor. } \\
\text { A: Acciones durante el desarrollo de la } \\
\text { actividad / tarea a realizar } \\
\text { CR: Actividades de cierre }\end{array}$ \\
\hline
\end{tabular}

Jornadas de Formación e Innovación Docente del Profesorado | № 2 (2019) Esta obra se distribuye con la licencia Creative Commons Reconocimiento-NoComercial-SinObraDerivada Internacional (CC BY-NC-ND 4.0.) 


\begin{tabular}{|l|l|l|}
\hline 1 & TP & $\begin{array}{l}\text { Publicación del guion de la práctica propuesta } \\
\text { (una semana antes de su comienzo) }\end{array}$ \\
\hline 2 & C & $\begin{array}{l}\text { Los alumnos deben resolver, antes de asistir } \\
\text { a la sesión práctica, un cuestionario en la } \\
\text { Plataforma de Enseñanza virtual sobre el guion } \\
\text { propuesto. }\end{array}$ \\
\hline 3 & TP & $\begin{array}{l}\text { Introducción a la sesión práctica y preguntas } \\
\text { clave. }\end{array}$ \\
\hline 4 & A & $\begin{array}{l}\text { Toma de medidas en las placas de desarrollo } \\
\text { proporcionadas por el profesor. }\end{array}$ \\
\hline 5 & TS & $\begin{array}{l}\text { Análisis de las medidas tomadas por los } \\
\text { alumnos }\end{array}$ \\
\hline 5 & CR & $\begin{array}{l}\text { Análisis crítico con el profesor y discusión de } \\
\text { los resultados obtenidos. El profesor resume } \\
\text { los aspectos más significativos de la sesión. }\end{array}$ \\
\hline
\end{tabular}

\section{Seguimiento de las actividades del Ciclo de Mejora en el Aula}

El seguimiento del rendimiento de los alumnos con los cambios introducidos se realizó alternando sesiones clásicas de prácticas, sin uso de la placa de desarrollo y con montaje de los circuitos, y sesiones en las que se usaron las placas de desarrollo, tal como se ha detallado en la secuencia de actividades.

Los alumnos entregaron dos memorias de prácticas para su evaluación. Una de ellas correspondía a las sesiones sin las placas de desarrollo propuestas y la otra a las sesiones que incorporaban la mejora descrita. Se compararon los resultados y se adaptaron los contenidos de las sesiones siguientes.

Para la evaluación de la labor de los alumnos en las sesiones prácticas se utilizó la ficha de la Tabla 2. La ficha recoge una serie de items que se evaluaron. Se tuvo en cuenta, tanto la realización de un test previo a la asistencia en el laboratorio como los propios resultados obtenidos en la práctica. Se valoró además la actitud de los 
alumnos en las sesiones prácticas y el trabajo en equipo realizado.

Tabla 2. Ficha de evaluación del alumnado

\begin{tabular}{|c|c|c|c|c|c|c|}
\hline Nombre & $\begin{array}{c}\text { Manejo } \\
\text { Instrumental }\end{array}$ & $\begin{array}{c}\text { Toma de } \\
\text { medidas }\end{array}$ & $\begin{array}{c}\text { Trabajo } \\
\text { previo }\end{array}$ & $\begin{array}{c}\text { Trabajo } \\
\text { en equipo }\end{array}$ & $\begin{array}{c}\text { Interpretación } \\
\text { de los } \\
\text { resultados }\end{array}$ & $\begin{array}{c}\text { Actitud } \\
\text { en el } \\
\text { laboratorio }\end{array}$ \\
\hline Alumno 1 & & & & & & \\
\hline$\ldots$. & & & & & & \\
\hline Alumno n & & & & & & \\
\hline
\end{tabular}

Se asignó una puntuación de 1 a 5 a cada uno de los alumnos, dependiendo de su desempeño en el laboratorio según la siguiente escala:

- 1-2: No se alcanzan los objetivos mínimos que se esperan del alumno.

- 3: Suficiente. El alumno ha adquirido los conocimientos elementales que se esperan.

- 4: Destacado. El alumno ha aprendido bien el manejo del instrumental y ha interiorizado los resultados de las medidas. Buena actitud en el trabajo en equipo y en el laboratorio.

- 5: Excelente. Se reserva esta calificación para los alumnos, que además de alcanzar el nivel anterior, sean capaces de modificar los circuitos propuestos para mejorar su comportamiento.

Estas puntuaciones se utilizaron para elaborar escaleras de aprendizaje que permitieron cuantificar el progreso de los alumnos en las sesiones prácticas con Placas de Desarrollo. En paralelo, se siguieron utilizando los mismos métodos de seguimiento que se han realizado otros años en la asignatura, como la interacción con el profesor durante las sesiones y la realización de un examen final. 


\section{Evaluación propuesta del CIMA y de la intervención}

Uno de los riesgos posibles del CIMA propuesto era que los alumnos perdieran destreza en el montaje de circuitos, al darse por hecho que en las sesiones en las que se usó la Placa de Desarrollo no tuvieron que realizar el montaje de circuitos. Se esperaba que esta posible carencia fuera compensada con un aumento generalizado del rendimiento del alumnado tomando medidas y manejando el instrumental del laboratorio. Se evaluaron estas dos competencias para validar el Ciclo de Mejora en el Aula.

El aprendizaje de los alumnos se evaluó mediante una memoria de prácticas en la que explicaron las medidas y el funcionamiento de los circuitos propuestos. Se comparó la calidad de las memorias entregadas con las de otros cursos en las que no se usaron Placas de Desarrollo.

La destreza de los alumnos utilizando el instrumental de laboratorio se evaluó por el profesor durante las propias sesiones. Se hicieron preguntas a los alumnos y se anotaron los resultados, con la idea de monitorizar el progreso del alumnado.

Finalmente, las opiniones del alumnado fueron tenidas en cuenta. Se les preguntó mediante un cuestionario anónimo sobre sus impresiones del CIMA.

\section{Aplicación del Ciclo de Mejora en el Aula}

\section{Relato resumido de las sesiones}

Como se comentó anteriormente, se alternaron las sesiones de prácticas que incorporaban las Placas de Desarrollo propuestas con sesiones en las que los alumnos 
debían montar los circuitos antes de realizar la toma de medidas. Nos centraremos en el análisis de las primeras, pues las segundas corresponden al enfoque clásico que se ha seguido en la asignatura durante años.

En la Figura 3, se muestra una de las Placas de Desarrollo que se utilizó en las sesiones, antes de que fuera montada, soldando los componentes electrónicos necesarios para su funcionamiento. Sobre ella, se colocaba una carátula distinta en cada práctica, similar a la que muestra en la Figura 3. Las placas son válidas para realizar múltiples experiencias, ya que se pueden reprogramar para que tengan funcionalidades distintas.
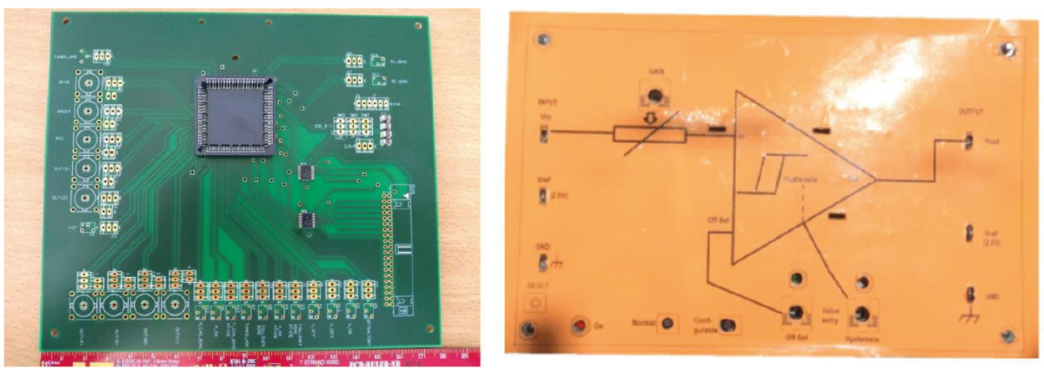

Figura 3. Foto de las placas de circuito impreso fabricadas y carátula de una de los experimentos que se programó en ella

En las primeras dos sesiones, las prácticas no fueron al ritmo esperado. Ello se debió a que tanto el alumnado como el profesorado no estaban habituados a utilizar las Placas de Desarrollo como herramienta docente. Se perdió algo de tiempo en explicar a los alumnos varias veces cómo proceder con ellas. El alumnado, habituado a realizar el propio montaje de sus circuitos, tuvo que entender que los circuitos ya se les daban montados en una placa de circuito impreso.

En las sesiones siguientes, se alcanzaron los objetivos propuestos. El alumnado consiguió finalizar a tiempo las prácticas, pudiendo dedicar más tiempo a la toma de medidas y al análisis de los resultados. 
Si comparamos las sesiones con Placas de Desarrollo con las sesiones en las que no se utilizaron, llegamos a la conclusión de que las placas de desarrollo resultaron ventajosas en las últimas sesiones en las que los circuitos que debían montarse eran más complejos y, por tanto, su montaje implicaba más tiempo al alumno. En las sesiones iniciales, los circuitos a montar eran más simples y, a parte, surgieron las complicaciones anteriormente mencionadas.

Hemos de destacar que existe una práctica en la que el montaje del circuito es especialmente complejo. Los alumnos siempre consumían la mayor parte de su tiempo con él, sin poder finalizarla. Este año, el uso de la Placas de Desarrollo permitió que se midiera en detalle este circuito, algo que nunca se había hecho antes en la asignatura.

En cuanto al alumnado, aunque en la primera sesión se mostró algo desconcertado con las Placas de Desarrollo, luego se habituó a ellas. Quizás, en las últimas sesiones, los alumnos confiaban en ellas en exceso, al percibirlas como una caja negra ideal que realizaba la función propuesta en la práctica.

\section{Evolución del aprendizaje de los estudiantes}

En la Tabla 3, se muestran las escaleras de aprendizaje del CIMA tras la evaluación del desempeño de los alumnos en el laboratorio en base al sistema de evaluación propuesto. Si se analizan los resultados, se aprecia una mejora tras la aplicación del CIMA. Destaca una reducción el número de alumnos que obtienen un rendimiento deficiente en las prácticas. La razón principal es que en las sesiones sin Placas de Desarrollo hay muchos alumnos que no logran montar el circuito propuesto. Ello hace que no puedan tomar las medidas propuestas en la práctica y no respondan ninguna de las cuestiones planteadas. Con las 
Placas de Desarrollo, se alivia esta situación, ya que se proporcionan los circuitos montados a los alumnos.

Tabla 3. Resultados obtenidos por los alumnos

\begin{tabular}{|c|c|c|} 
& \multicolumn{1}{c}{ Sin Placas Desarrollo } & Uso Placas de Desarrollo \\
Nivel 5: Excelente & $7 \%$ & $9 \%$ \\
\cline { 2 - 3 } Nivel 4: Destacado & $16 \%$ & $22 \%$ \\
\cline { 2 - 3 } Nivel 3: Suficiente & $51 \%$ & $64 \%$ \\
\cline { 2 - 3 } Nivel 1-2: Deficiente & $\mathbf{2 6 \%}$ & $5 \%$ \\
\cline { 2 - 3 }
\end{tabular}

En el resto de niveles de la escalera de aprendizaje la diferencia no es tan significativa, sobretodo, en los niveles altos. En general, los alumnos que manejan bien el instrumental de laboratorio tienen un buen desempeño en las dos situaciones.

\section{Evaluación del CIMA puesto en práctica}

\section{Cuestiones a mantener y cambios a introducir en un futuro CIMA}

Hemos de destacar, que uno de los aspectos que se evaluó para elaborar las escaleras de aprendizaje fue el manejo del instrumental de laboratorio. La sensación del profesorado es que esta competencia se desarrolla menos con el uso de la Placas de Desarrollo, ya que se dedica menos tiempo al montaje de circuitos. Pensamos que esta carencia del CIMA debe complementarse con sesiones en las que no se haga uso de las Placas de Desarrollo, ya que éstas limitan el tiempo que se dedica a montar circuitos.

Las Placas de Desarrollo se han mostrado especialmente útiles en las prácticas en las que el montaje de circuitos era especialmente complejo. En base a ello, se propone para cursos venideros que solo se utilicen dichas 
herramientas en la fase final de la asignatura, en la cual ya los alumnos han adquirido la destreza necesaria para montar circuitos. Se comprobó que el alumnado y el profesorado necesitó una toma de contacto con las Placas de Desarrollo. Se propone pues que exista una sesión específica dedicada a su manejo, con la idea de que la primera sesión de prácticas evaluables con ellas no sea improductiva.

En cuanto al Mapa de Contenidos de la asignatura y el sistema de evaluación propuesto, no se proponen cambios futuros; ambos han demostrado ser adecuados para conseguir los objetivos de la asignatura.

\section{Aspectos de la experiencia a incorporar en la docencia habitual}

Las Placas de Desarrollo diseñadas han demostrado ser una poderosa herramienta pedagógica cuando no se dispone tiempo en el laboratorio para la puesta en funcionamiento de circuitos relativamente complejos. Dado que los nuevos planes de estudio limitan mucho la duración de las sesiones de laboratorio, pretendemos que se incorporen a la docencia del Departamento de forma progresiva, habiendo varias asignaturas con prácticas que son buenas candidatas.

Hemos de destacar que las Placas de Desarrollo son enteramente programables. Una misma placa puede ser programada para que sobre ella se realicen prácticas distintas de distintas asignaturas. En colaboración con profesores de la Universidad de Cádiz, que iniciaron este proyecto, se están elaborando nuevas experiencias que puedan ser trasladadas al aula. Las propias Placas de Desarrollo han sido sometidas a varias fases de mejora y adecuación a la experiencia en las aulas. El objetivo final es pues doble: por una parte, intensificar su uso en el aula. 
Por otra, aumentar el número de experimentos docentes que puedan ser implementados en ellas, con la idea de tener una biblioteca de experiencias que puedan compartir docentes de varias facultades.

\section{Principios didácticos que han guiado la experiencia y que deben permanecer en el futuro}

Los principios didácticos que han fundamentado la innovación docente en el proceso de enseñanza-aprendizaje y que deben permanecer en la asignatura son los siguientes:

El aprendizaje basado en la realización de experiencias prácticas, cercanas a la realidad y continuadas en el tiempo, es más efectivo y perdura más que un aprendizaje a ráfagas, basado en interminables días y noches de estudio intenso, unos días antes del examen (Finkel, 2008).

La adopción de un enfoque constructivista (Delval, 1997), según el cual el alumno es el verdadero protagonista de su aprendizaje, teniendo éste un papel activo en el que no sólo reproduce los contenidos facilitados por el docente, pero además, evalúa continuamente y somete a crítica de forma continua sus propios conocimientos (Gómez Rijo, 2010).

La creación de un entorno de trabajo y de un entorno enriquecedor que lleve al alumno a trabajar de forma continuada y autónoma, y a plantearse interrogantes sobre los conceptos impartidos, y a aprender por sí mismos (Bain, 2007). El uso de las Placas de Desarrollo propuestas permite que los alumnos progresen por sí mismos, sin necesidad de que el profesor tenga que verificar el montaje de sus circuitos antes de realizar la toma de medidas.

Jornadas de Formación e Innovación Docente del Profesorado | № 2 (2019) Esta obra se distribuye con la licencia Creative Commons Reconocimiento-NoComercial-SinObraDerivada 
La elección contenidos de interés para los estudiantes y que estén relacionados con los dispositivos electrónicos que usan a diario. Este hecho tradicionalmente ha dado buenos resultados pedagógicos (Ausubel, 1978).

\section{Conclusiones}

Se han evaluado las bondades de una herramienta docente que permite optimizar el uso del tiempo en las sesiones de prácticas de Electrónica. Consiste en una Placa de Desarrollo multipropósito que permite programar e implementar sobre ella distintos circuitos electrónicos de interés para su análisis en el laboratorio. La herramienta posibilita que el alumno se centre en la toma de medidas y su análisis, evitando que se desvie la atención en la puesta a punto del instrumental y el montaje de los circuitos bajo estudio. Hemos identificado situaciones en las que las Placas de Desarrollo son particularmente útiles. Se pretende extender su uso en el aula en tales casos de interés.

Jornadas de Formación e Innovación Docente del Profesorado I № 2 (2019) Esta obra se distribuye con la licencia Creative Commons 


\section{Bibliografía}

Porlan, R et al. (2017). Enseñanza Universitaria. Cómo mejorarla (2a edición). Madrid: Morata.

Finkel, D. (2008). Dar clase con la boca cerrada. Valencia: Publicaciones de la Universidad de Valencia.

Ausubel, D. Novak, J., y Hanesian, H. (1978). Educational Psychology: A Cognitive View (2nd Ed.). New York: Holt, Rinehart \& Winston.

Delval, J. (1997). Tesis sobre el constructivismo. En Rodrigo, M.J. y Arnay J., La construcción del conocimiento escolar (pp. 15-35). Barcelona: Paidós.

Gómez Rijo, A. (2010). El alumnado como constructor de su propio aprendizaje en el área de educación fisica. Huelva: Universidad de Huelva, 52-61, 6.

Bain, K. (2006). Lo que hacen los mejores profesores universitarios, Valencia: Publicaciones de la Universidad de Valencia. 